\title{
ON TOPOLOGICAL ENTROPY OF TRIANGULAR MAPS OF THE SQUARE
}

\author{
Lluís Alsedà, Sergi ́ F. Kolyada and Lubomír Snoha
}

\begin{abstract}
We study the topological entropy of triangular maps of the square. We show that such maps differ from the continuous maps of the interval because there exist triangular maps of the square of "type $2^{\infty}$ " with infinite topological entropy. The set of such maps is dense in the space of triangular maps of "type at most $2^{\infty}$ " and the topological entropy as a function of the triangular maps of the square is not lower semicontinuous. However, we show that for these maps the characterisation of the lower bounds of the topological entropy depending on the set of periods is the same as for the continuous maps of the interval.
\end{abstract}

\section{INTRODUCtion AND STATEMENT OF THE MAIN RESUltS}

For a compact topological space $X$, denote by $\mathcal{C}(X, X)$ the set of all continuous self-maps of $X$. The dynamical system given by a map $G \in \mathcal{C}(X, X)$ is called topologically chaotic or simple if the topological entropy $h(G)$ of $G$ is positive or zero, respectively. A natural question is: which properties characterise the topologically chaotic dynamical systems?

If $I$ is a compact interval of the real line and $f \in \mathcal{C}(I, I)$, then the topological entropy of $f$ is zero if and only if the period of any periodic point of $f$ is a power of two (see $[7,21])$. To state this result in other words, denote by $\mathbb{N}$ the set of all positive integers and introduce the Sharkovskii ordering, $>$ on the set $\mathbb{N} \cup\left\{2^{\infty}\right\}$ by:

$$
\begin{aligned}
& 3,>5,>7,>\ldots,>2 \cdot 3,>2 \cdot 5,>2 \cdot 7,>\ldots,>4 \cdot 3,>4 \cdot 5,>4 \cdot 7,>\ldots,>\ldots \\
& ,>2^{n} \cdot 3 .>2^{n} \cdot 5,>2^{n} \cdot 7,>\ldots,>2^{\infty},>\ldots,>2^{n},>\ldots,>16,>8,>4,>2,>1 .
\end{aligned}
$$

We shall also use the symbol,$\geqslant$ in the natural way. For $s \in \mathbb{N} \cup\left\{2^{\infty}\right\}$ we denote by $S(s)$ the set $\{k \in \mathbb{N}: s, \geqslant k\}\left(S\left(2^{\infty}\right)\right.$ stands for the set $\left.\left\{1,2,4, \ldots, 2^{k}, \ldots\right\}\right)$ and by

Received 23rd July, 1992

The authors thank the Institute of Mathematics of the Ukrainian Academy of Sciences and the Centre de Recerca Matemàtica, co organisers of the Soviet-Czechoslovak-Spanish summer school on Dynamical Systems (Kiev, 1991), where this work was started. They also thank the organisers of ECIT 91 (Lisboa) for the opportunity given to Ll. Alsedà and $\dot{L}$. Snoha to meet and discuss this paper. Ll. Alsedà was partially supported by the DGICYT grant number PB90-0695.

Copyright Clearance Centre, Inc. Serial-fee code: 0004-9729/93 \$A2.00+0.00. 
Per $(f)$ we denote the set of periods of all periodic points of $f$. The Sharkovskil theorem [22] says that for every $f \in \mathcal{C}(I, I)$ there exists $s \in \mathbb{N} \cup\left\{2^{\infty}\right\}$ such that $\operatorname{Per}(f)=S(s)$. Conversely, for every $s \in \mathbb{N} \cup\left\{2^{\infty}\right\}$ there exists $f \in \mathcal{C}(I, I)$ with $\operatorname{Per}(f)=S(s)$. If $\operatorname{Per}(f)=S(s)$, then $f$ is said to be of type $s$. When speaking of types we consider them to be ordered by the Sharkovskiu ordering.

It is known (see [21]) that if $f$ is an interval map then $h(f)=0$ if and only if $f$ is of type at most $2^{\infty}$. Thus, the maps of type $2^{\infty}$ are the boundary between topologically chaotic and simple maps on the interval. All these maps are simple.

More about the topological dynamics of one-dimensional maps can be found in $[2,4,23]$. Recently, necessary and sufficient conditions for the topological entropy to be positive in terms of the existence of periodic orbits of certain kinds were found for the continuous self-maps of graphs (see [6, 20]) and for the orientation preserving diffeomorphisms of two-dimensional discs (see, for example, $[11,19]$ ).

In the present paper we study the topological entropy of triangular maps on the square. We recall that if $I$ is a compact interval of the real line, then a continuous map $F$ from $I^{2}$ into itself is called triangular if $F(x, y)=(f(x), g(x, y))$, that is, if the first coordinate of the image of a point depends only on the first coordinate of that point. Here $f: I \rightarrow I$ and $g: I^{2} \rightarrow I$ are continuous. We can also write $F(x, y)=\left(f(x), g_{x}(y)\right)$ where $g_{x}: I \rightarrow I$ is a family of continuous maps depending continuously on $x$. The set of all continuous triangular maps from $I^{2}$ into itself will be denoted by $\mathcal{C}_{\Delta}\left(I^{2}, I^{2}\right)$.

For $f, \varphi \in \mathcal{C}(I, I)$ let $d_{1}(f, \varphi)=\max _{x \in I}|f(x)-\varphi(x)|$ and if $F(x, y)=\left(f(x), g_{x}(y)\right)$ and $\Phi(x, y)=\left(\varphi(x), \psi_{x}(y)\right)$ are maps from $\mathcal{C}_{\Delta}\left(I^{2}, I^{2}\right)$ we set

$$
d_{2}(F, \Phi)=\max _{(x, y) \in I^{2}} \max \left\{|f(x)-\varphi(x)|,\left|g_{x}(y)-\psi_{x}(y)\right|\right\}
$$

Then, $\left(\mathcal{C}(I, I), d_{1}\right)$ and $\left(\mathcal{C}_{\Delta}\left(I^{2}, I^{2}\right), d_{2}\right)$ are metric spaces. We shall have this in mind whenever we consider the sets $\mathcal{C}(I, I)$ and $\mathcal{C}_{\Delta}\left(I^{2}, I^{2}\right)$ as spaces.

It is known (see [14]) that the characterisation of the set of periods for maps from $\mathcal{C}_{\Delta}\left(I^{2}, I^{2}\right)$ coincides with the one for maps from $\mathcal{C}(I, I)$. That is, for every $F \in \mathcal{C}_{\Delta}\left(I^{2}, I^{2}\right)$ there exists $s \in \mathbb{N} \cup\left\{2^{\infty}\right\}$ such that $\operatorname{Per}(F)=S(s)$. Conversely, for every $s \in \mathbb{N} \cup\left\{2^{\infty}\right\}$ there exists $F \in \mathcal{C}_{\Delta}\left(I^{2}, I^{2}\right)$ such that $\operatorname{Per}(F)=S(s)$. As in the case of interval maps we say that a triangular map $F$ has type $s$ if $\operatorname{Per}(F)=S(s)$. On the other hand, from [15] (see also [16]), we know that if $F \in \mathcal{C}_{\Delta}\left(I^{2}, I^{2}\right)$ is of type greater than $2^{\infty}$ then $h(F)>0$; if it is of type less than $2^{\infty}$ then $h(F)=0$ and if $F$ is of type $2^{\infty}$ then both cases are possible. In fact, there exist examples of maps from $\mathcal{C}_{\triangle}\left(I^{2}, I^{2}\right)$ of type $2^{\infty}$ with topological entropy zero and with positive entropy. Thus, the dynamics of triangular maps of type $2^{\infty}$ is more complicated than that of interval maps of type $2^{\infty}$. In connection with this problem the following natural question was 
proposed by J. Llibre at the Soviet-Czechoslovak-Spanish summer school on Dynamical Systems. Does there exist $k<\infty$ such that $h(F) \leqslant k$ whenever $F \in \mathcal{C}_{\Delta}\left(I^{2}, I^{2}\right)$ is of type $2^{\infty}$ ? The negative answer to this question is a consequence of the following result.

Theorem A. There is a map $\Phi \in \mathcal{C}_{\Delta}\left(I^{2}, I^{2}\right)$, of type $2^{\infty}$, with $h(\Phi)=\infty$.

Moreover, there are a lot of such maps, since we have

THEOREM B. The set of all maps from $\mathcal{C}_{\Delta}\left(I^{2}, I^{2}\right)$ of type $2^{\infty}$ with infinite topological entropy is dense in the space of all maps from $\mathcal{C}_{\Delta}\left(I^{2}, I^{2}\right)$ of type at most $2^{\infty}$.

The next theorem shows again that there are substantial differences between triangular and interval maps. Topological entropy on the interval is lower semi-continuous but this is not the case for triangular maps.

THEOREM C. The topological entropy, as a function of the continuous triangular maps of the square, is not lower semi-continuous.

However, a very important property of the topological entropy of interval maps can be extended to triangular maps on the square. Namely, the characterisation of the lower bounds of topological entropy depending on the set of periods (see $[5,12,13])$. To state this result we have to introduce some notation.

Let $\lambda_{q}$ be the largest root of the polynomial $x^{q}-2 x^{q-2}-1$, where $q>1$ is odd. Then, if $n=2^{m} \cdot q$ with $m \geqslant 0$ and $q \geqslant 1$ odd we set

$$
\tau_{n}= \begin{cases}1 & \text { if } q=1 \\ \lambda_{q}^{2^{-m}} & \text { if } q>1\end{cases}
$$

The following result shows that the characterisation of the lower bounds of the topological entropy for triangular maps is the same as for interval maps.

TheOREm D. Let $F \in \mathcal{C}_{\Delta}\left(I^{2}, I^{2}\right)$ have a periodic orbit of period $n$. Then $h(F) \geqslant \log \tau_{n}$. Conversely, for each $n \in \mathbb{N}$ there exists a map $F_{n} \in \mathcal{C}_{\Delta}\left(I^{2}, I^{2}\right)$ of type $n$ such that $h\left(F_{n}\right)=\log \tau_{n}$.

REMARK. From the proof of Theorem D, we see that this result is also valid in the general case of triangular maps on $I^{n}$ for any $n$.

The paper is organised as follows. In Section 2 we introduce some notation and we state some known preliminary results. In Section 3 we prove Theorems A and B. In the very short Section 4 we prove Theorem $\mathrm{C}$ and, lastly, in Section 5 we prove Theorem $\mathrm{D}$.

\section{DEFINITIONS AND PRELIMINARY RESULTS}

We start by noting that, without loss of generality, we can assume that $I=[0,1]$ and we shall do so in the rest of the paper for simplicity. 
If $F=\left(f, g_{x}\right)$ is a map from $\mathcal{C}_{\Delta}\left(I^{2}, I^{2}\right)$ then $f$ is called its basis map. For $x \in I$ we define the fibre on $x$ by $I_{x}=\{x\} \times I$. Denote by pr: $I^{2} \longrightarrow I$ the first projection, that is, if $(x, y) \in I^{2}$ and $A \subset I^{2}$ then $\operatorname{pr}((x, y))=x$ and $\operatorname{pr}(A)=\left\{x \in I: I_{x} \cap A \neq \emptyset\right\}$.

Now let $X$ be a compact metric space and let $f \in \mathcal{C}(X, X)$. The iterates of $f$ are defined inductively by $f^{0}=$ id and $f^{n+1}=f \circ f^{n}, n \geqslant 0$. A point $x \in X$ is periodic if $f^{n}(x)=x$ for some $n>0$. The least such $n$ is called the period of $x$. A point of period one is called a fixed point. The orbit of $x \in X$ under $f$ is $\operatorname{Orb}(f, x)=\left\{f^{n}(x): n \geqslant 0\right\}$. Similarly, for $A \subset X$ we put $\operatorname{Orb}(f, A)=\bigcup_{n=0}^{\infty} f^{n}(A)$. If $x$ is periodic with period $n$ then its orbit is called a periodic orbit with period $n$. The set of periodic points of a map $f \in \mathcal{C}(X, X)$ will be denoted by $P(f)$.

Now we recall the definition of topological entropy. Let $X$ be a compact topological space and let $G \in \mathcal{C}(X, X)$. If $\mathcal{A}$ is a family of subsets of $X$ then we set

$$
\mathcal{A}^{n}=\mathcal{A}_{G}^{n}=\left\{\bigcap_{i=0}^{n-1} G^{-i}\left(A_{i}\right): A_{i} \in \mathcal{A} \text { for } i=0, \ldots, n-1 \text { and } \bigcap_{i=0}^{n-1} G^{-i}\left(A_{i}\right) \neq \emptyset\right\}
$$

If $\mathcal{A}$ is an open cover of $X$, denote by $\mathcal{N}(\mathcal{A})$ the minimal possible cardinality of a subcover chosen from $\mathcal{A}$. Then

$$
h(G, \mathcal{A})=\lim _{n \rightarrow \infty} \frac{1}{n} \log \mathcal{N}\left(\mathcal{A}_{G}^{n}\right)
$$

is the topological entropy of $G$ on the cover $\mathcal{A}$. The topological entropy of $G$ is then defined by (see [1]):

$$
h(G)=\sup \{h(G, \mathcal{A}): \mathcal{A} \text { is an open cover of } X\}
$$

We shall also use Bowen's definition of topological entropy (see $[\mathbf{8}, \mathbf{1 0}]$ ), which is equivalent to the above one. Let $(X, \rho)$ be a compact metric space and let $G \in \mathcal{C}(X, X)$. A subset $E$ of $X$ is called $(n, \varepsilon)$-separated if for every two different points $x, y \in E$ there exists $0 \leqslant j<n$ with $\rho\left(G^{j}(x), G^{j}(y)\right)>\varepsilon$. A set $E_{1} \subset X(n, \varepsilon)$-spans another set $K \subset X$ provided that for each $x \in K$ there is $y \in E_{1}$ for which $\rho\left(G^{j}(x), G^{j}(y)\right) \leqslant \varepsilon$ for all $0 \leqslant j<n$.

For a compact set $K \subset X$ let $r_{n}(\varepsilon, K)$ be the minimal possible cardinality of a set $E_{1}$ which $(n, \varepsilon)$-spans $K$ and let $s_{n}(\varepsilon, K)$ be the maximal possible cardinality of an $(n, \varepsilon)$-separated set $E$ contained in $K$ (we shall write $r_{n}(\varepsilon, K, G)$ and $s_{n}(\varepsilon, K, G)$ if we wish to stress the dependence on $G$ ). Finally define

$$
r(\varepsilon, K, G)=\limsup _{n \rightarrow \infty} \frac{1}{n} \log r_{n}(\varepsilon, K, G)
$$


and

Then we set

$$
s(\varepsilon, K, G)=\limsup _{n \rightarrow \infty} \frac{1}{n} \log s_{n}(\varepsilon, K, G) .
$$

$$
\begin{aligned}
h_{\rho}(G, X) & =\lim _{\varepsilon \rightarrow 0} s(\varepsilon, K, G) \\
& =\lim _{\varepsilon \rightarrow 0} r(\varepsilon, K, G)
\end{aligned}
$$

and (see $[9,10])$

$$
h(G)=h_{\rho}(G, X) .
$$

We shall often use the well known formula $h\left(G^{n}\right)=n \cdot h(G), n \geqslant 0$.

For $F \in \mathcal{C}_{\Delta}\left(I^{2}, I^{2}\right)$ set $h_{f}(F)=\sup _{x \in I} h\left(F, I_{x}\right)$. Then, from Bowen's formula (see Theorem 17 in [8]; see also [16]) one gets

$$
h(f)+h_{f}(F) \geqslant h(F) \geqslant \max \left\{h(f), h_{f}(F)\right\} .
$$

So, if the basis map $f$ of $F$ is simple, that is, of type at most $2^{\infty}$, then $h(F)=h_{f}(F)$.

If $X_{1}, X_{2}$ are compact topological spaces, $f \in \mathcal{C}\left(X_{1}, X_{1}\right)$ and $g \in \mathcal{C}\left(X_{2}, X_{2}\right)$ then $f, g$ are said to be topologically conjugate if there is a homeomorphism $\varphi: X_{1} \rightarrow X_{2}$ such that $g \circ \varphi=\varphi \circ f$. In such a case $h(f)=h(g)$ and $\operatorname{Per}(f)=\operatorname{Per}(g)$. A set $A$ is called $f$-invariant if $f(A) \subset A$. The closure of $A$ is denoted by $\mathrm{Cl}(A)$.

\section{TRIANGULAR MAPS OF TYPE $2^{\infty}$ WITH INFINITE ENTROPY}

We have mentioned in the introduction that in [15] it was proved that there exist continuous triangular maps of the square of type $2^{\infty}$ with positive topological entropy. Now we are going to strengthen this result by proving Theorems $\mathrm{A}$ and $\mathrm{B}$.

Proof of Theorem A: Let $F:(x, y) \mapsto\left(f_{*}(x), g(x, y)\right)$ be the continuous triangular map of type $2^{\infty}$ with positive topological entropy from [15] (see also [16]). So $f_{*}(x)=\lambda_{*} x(1-x)$ is the logistic unimodal map of type $2^{\infty}, g(x, 0)=g(x, 1)=0$ for every $x$ and $h(F)>0$ is finite.

For every positive integer $n, F^{n}$ is a continuous triangular map and so, there is a map $h_{n}$ such that $F^{n}(x, y)=\left(f_{*}^{n}(x), h_{n}(x, y)\right)$. Clearly, $h_{n}(x, 0)=h_{n}(x, 1)=0$.

Take a sequence of points $0=a_{1}<b_{1}<a_{2}<b_{2}<\cdots<a_{n}<b_{n}<\cdots$ converging to 1 . We are going to construct a map $\Phi=\left(\varphi, \psi_{x}\right)$ from $\mathcal{C}_{\Delta}\left(I^{2}, I^{2}\right)$ of type $2^{\infty}$ with $h(\Phi)=\infty$. First, define the basis map $\varphi \in \mathcal{C}(I, I)$ such that $\left.\varphi\right|_{\left[a_{n}, b_{n}\right]}=\sigma_{n}^{-1} \circ f_{*}^{n} \circ \sigma_{n}$ for each $n \geqslant 1$, where $\sigma_{n}$ is the unique increasing affine map from $\left[a_{n}, b_{n}\right]$ onto $[0,1]$ and $\left.\varphi\right|_{\left[b_{n}, a_{n+1}\right]}$ is affine for each $n \geqslant 1$.

Clearly $\varphi$ is of type $2^{\infty}$ since, for each $n=1,2 \ldots$, it has no periodic points in the interval $\left(b_{n}, a_{n+1}\right)$ and $\left.\varphi\right|_{\left[a_{n}, b_{n}\right]}$ is topologically conjugate to $f_{*}^{n}$.

It remains to define the maps $\psi_{x}$ for $x \in I$. If $x \in\left[a_{n}, b_{n}\right]$ with $n=1,2, \ldots$, then we define $\psi_{x} \in \mathcal{C}(I, I)$ such that $\psi_{x}(y)=\sigma_{n}^{-1}\left(h_{n}\left(\sigma_{n}(x), \sigma_{n}(y)\right)\right)$ for $y \in$ 
$\left[a_{n}, b_{n}\right], \psi_{x}(0)=0, \psi_{x}(1)=1$ and $\left.\psi_{x}\right|_{\left[0, a_{n}\right]}$ and $\left.\psi_{x}\right|_{\left[b_{n}, 1\right]}$ are affine. Set also $\psi_{1}=$ id. Finally, for $x \in\left[b_{n}, a_{n+1}\right]$ with $n \geqslant 1$, let $\psi_{x}$ be any family of continuous maps depending continuously on $x$ such that $\Phi$ is continuous. Say:

$$
\psi_{x}(y)=\frac{x-b_{n}}{a_{n+1}-b_{n}} \psi_{a_{n+1}}(y)+\frac{a_{n+1}-x}{a_{n+1}-b_{n}} \psi_{b_{n}}(y)
$$

It is clear that $\Phi=\left(\varphi, \psi_{x}\right)$ belongs to $\mathcal{C}_{\Delta}\left(I^{2}, I^{2}\right)$ and that, by construction, $\left.\Phi\right|_{\left[a_{n}, b_{n}\right]^{2}}$ is topologically conjugate to $F^{n}$. Since $h\left(F^{n}\right)=n h(F), h(F)>0$, and $F^{n}$ is of type $2^{\infty}$, we get that $h(\Phi)=\infty$ and $\Phi$ is of type at least $2^{\infty}$. On the other hand, it is easy to see that every periodic point of $\Phi$ with the first coordinate lying in $\bigcup_{n=1}^{\infty}\left[a_{n}, b_{n}\right] \cup\{1\}$ has period a power of two. Further, if $x \in \bigcup_{n=1}^{\infty}\left(b_{n}, a_{n+1}\right)$ then $x$ is not a periodic point of $\varphi$ and so $I_{x}$ contains no periodic point of $\Phi$. Therefore $\Phi$ is of type $2^{\infty}$. This ends the proof of the theorem.

To prove Theorem B we need some auxiliary results.

Lemma 3.1. Let $f \in \mathcal{C}(I, I)$ have no periodic orbits with odd periods greater than 1. Let $c$ be a fixed point of $f$ and suppose that for some $\delta>0, f(x)>x$ for all $x \in(c, c+\delta]$. Then there exists $0<\Delta<\delta$ such that

(i) $f([c, c+\Delta]) c[c, c+\delta]$.

(ii) For each $x \in(c, c+\Delta)$ there exists an $n(x)$ such that $x<f(x)<$ $f^{2}(x)<\cdots<f^{n(x)}(x)$ and $f^{n(x)}(x) \geqslant c+\Delta$.

(iii) $\operatorname{Orb}(f,[c+\Delta, c+\delta]) \subset[c+\Delta, 1]$.

(iv), If $g \in \mathcal{C}(I, I)$ maps $(c, c+\Delta)$ into $[c, c+\delta]$ and $g(x)=f(x)$ for every $x \in I \backslash(c, c+\Delta)$, then $P(g)=P(f) \cup\{x \in P(g): \operatorname{Orb}(g, x) \subset(c, c+\Delta)\}$.

Proof: Take the least $\delta_{1}>0$ such that $f\left(c+\delta_{1}\right)=c+\delta$ and $0<\Delta<\delta_{1}$ such that $f(c+\Delta)=c+\delta_{1}$. Then (i) holds trivially. Let $x \in(c, c+\Delta)$ and consider the sequence $\left\{f^{n}(x)\right\}_{n=0}^{\infty}$. If $f^{n}(x)<c+\Delta$ for all $n$, since $f(x)>x$ for each $x \in(c, c+\delta]$, we get that the sequence is increasing. Thus, there exists $\alpha=\lim _{n \rightarrow \infty} f^{n}(x)$ and $\alpha \leqslant c+\Delta$. Of course, $\alpha=f(\alpha)$; a contradiction. This ends the proof of (ii). To prove (iii) we claim that $\operatorname{Orb}\left(f,\left[c+\delta_{1}, c+\delta\right]\right) \subset[c+\Delta, 1]$. Suppose on the contrary that for some $z \in\left[c+\delta_{1}, c+\delta\right]$ and some positive integer $k$ we have $f^{k}(z)<c+\Delta$. Clearly, $k \geqslant 2$. Now take $z_{1} \in\left[c+\Delta, c+\delta_{1}\right]$ with $f\left(z_{1}\right)=z$. Then either for $x=z$ and $n=k$ or for $x=z_{1}$ and $n=k+1$ we have that $n$ is odd and $f^{n}(x)<x<f(x)$. But this implies (see [18, Proposition 2.2]) that $f$ has a periodic point of an odd period greater than 1 ; a contradiction. So $\operatorname{Orb}\left(f,\left[c+\delta_{1}, c+\delta\right]\right) \subset[c+\Delta, 1]$ and, thus, (iii) holds. Finally, from (i)-(iii) we get that $P(f) \cap(c, c+\Delta)=\emptyset$. Hence, (iv) is satisfied. 
In the sequel, the interval $[c, c+\Delta)$ from the lemma will be called an $f$-repulsive right-hand side neighbourhood of $c$ corresponding to $[c, c+\delta]$. It is easy to see that for any $0<\Delta^{\prime} \leqslant \Delta,\left[c, c+\Delta^{\prime}\right)$ is also an $f$-repulsive right-hand side neighbourhood of $c$ corresponding to $[c, c+\delta]$. Since an analogous result holds if $f(x)<x$ for all $x \in$ $[c-\delta, c)$, in the sequel we shall also talk about $f$-repulsive left-hand side neighbourhoods of $c$ corresponding to $[c-\delta, c]$.

Lemmma 3.2. Let $f, g \in \mathcal{C}(I, I)$ and let $c$ be a fixed point of $f$. Let $f$ have no periodic orbits with odd period greater than 1. Then, for each $\varepsilon, \eta>0$ there exist an open interval $L \subset I$ such that $c \in \mathrm{Cl}(L)$ and the diameter of $L$ is smaller than $\eta$; a closed interval $K \subset L$ such that $c \notin K$ and a map $\tilde{f} \in \mathcal{C}(I, I)$ satisfying the following conditions:

(i) $\left.f\right|_{(I \backslash L) \cup\{c\}}=\left.\tilde{f}\right|_{(I \backslash L) \cup\{c\}}$.

(ii) $\left.\tilde{f}\right|_{K}$ is topologically conjugate to $g$.

(iii) Each periodic orbit of $\tilde{f}$ is contained either in $K$ or in $L \backslash K$ or in $I \backslash L$.

(iv) $\tilde{f}$ has at most three periodic points in $L \backslash K$ and they have period at most 2.

(v) $d_{1}(f, \tilde{f})<\varepsilon$

Proof: We first consider the case $c \neq 1$. Choose $0<\delta<\min \{\varepsilon / 2, \eta / 2,1-c\}$ such that $|f(x)-c|<\varepsilon / 2$ for all $x \in[c-\delta, c+\delta] \cap I$. We consider three cases:

CASE 1. There exists a point $b \in(c, c+\delta]$ such that $c \leqslant f(b) \leqslant b$. Then we set $L=(c, b)$ and we choose a closed interval $K \subset L$ and points $d \in(c, \min K)$ and $d^{\prime} \in(\max K, b)$. We define $\tilde{f} \in \mathcal{C}(I, I)$ such that it satisfies conditions (i) and (ii), $\tilde{f}(x)=\tilde{f}(\min K)$ for each $x \in[d, \min K], \tilde{f}\left(d^{\prime}\right)=c$ and $\tilde{f}$ is affine on each of the intervals $[c, d],\left[\max K, d^{\prime}\right]$ and $\left[d^{\prime}, \sup L\right]$. Then, $\tilde{f}(\mathrm{Cl}(L)) \subset \mathrm{Cl}(L)$ and $\tilde{f}$ has no periodic points in $L \backslash K$. Thus, $\tilde{f}$ is the desired map.

CASE 2. $f(x)>x$ for each $x \in(c, c+\delta]$. By Lemma 3.1 there exists an $f$-repulsive right-hand side neighbourhood $[c, c+\Delta)$ of $c$ corresponding to $[c, c+\delta]$. Then we set $L=(c, c+\Delta)$ and we define $\tilde{f}$ as in Case 1 . Then, $\tilde{f}$ has no periodic orbit contained in $L \backslash K$ except for a fixed point in $\left(d^{\prime}, c+\Delta\right)$. Thus, in view of Lemma 3.1, $\tilde{f}$ satisfies the remaining conditions from the statement of the lemma.

CASE 3. $f(x)<c$ for each $x \in(c, c+\delta]$. Then, $c \neq 0$ and, without loss of generality, we may assume that $0 \leqslant c-\delta$. If $f(x) \leqslant c$ for some $x \in[c-\delta, c)$ then we can proceed in a similar way to Cases 1 and 2 (but now one has to take $L$ such that $c=\sup L$ instead of $c=\inf L$ ). Therefore, we may assume that $f(x)>c$ for each $x \in[c-\delta, c)$. Take $0<\delta_{1}<\delta$ such that $f\left(\left[c-\delta_{1}, c+\delta_{1}\right]\right) \subset[c-\delta, c+\delta]$. Then, $f^{2}(x)>c$ for all $x \in\left(c, c+\delta_{1}\right]$ and $f^{2}(x)<c$ for all $x \in\left[c-\delta_{1}, c\right)$. We consider two subcases. 
SUBCASE 3A. Either $f^{2}(z) \leqslant z$ for some $z \in\left(c, c+\delta_{1}\right]$ or $f^{2}\left(z^{\prime}\right) \geqslant z^{\prime}$ for some $z^{\prime} \in\left[c-\delta_{1}, c\right)$. We shall only consider the first possibility. In the second one we can construct $\tilde{f}$ in a similar way. We take $L=(f(z), z)$, a closed interval $K \subset(c, z)$, $d \in(c, \min K)$ and $d^{\prime} \in(\max K, z)$. We define $\tilde{f} \in \mathcal{C}(I, I)$ satisfying conditions (i) and (ii), $\tilde{f}(x)=\tilde{f}(\min K)$ for each $x \in[d, \min K], \tilde{f}\left(d^{\prime}\right)=c$ and $\tilde{f}$ is affine on each of the intervals $[f(z), c],[c, d],\left[\max K, d^{\prime}\right]$ and $\left[d^{\prime}, z\right]$. Clearly, $\tilde{f}(\mathrm{Cl}(L)) \subset \mathrm{Cl}(L)$. Moreover, it can be seen that $\{c\}$ is the only periodic orbit of $\tilde{f}$ lying in $L \backslash K$. Thus, $\tilde{f}$ is the desired map.

SUBCASE 3B. $f^{2}(x)>x$ for each $x \in\left(c, c+\delta_{1}\right]$ and $f^{2}(x)<x$ for each $x \in$ $\left[c-\delta_{1}, c\right)$. Take $0<\Delta<\delta_{1}$ such that $f([c-\Delta, c+\Delta]) \subset\left[c-\delta_{1}, c+\delta_{1}\right]$ and $[c, c+\Delta)$ and $(c-\Delta, c]$ are $f^{2}$-repulsive neighbourhoods of $c$ corresponding to $\left[c, c+\delta_{1}\right]$ and $\left[c-\delta_{1}, c\right]$, respectively. By Lemma 3.1, we have that the interval $[c-\Delta, c+\Delta]$ is not $f^{2}$ invariant and so it is not $f$-invariant. This implies that there exists either $x \in[c-\Delta, c)$ with $f(x)>c+\Delta$ or $x \in(c, c+\Delta]$ with $f(x)<c-\Delta$. We shall only consider the first possibility. The proof of the lemma in the other case follows similarly.

We set $a_{1}=\max \{x \in[c-\Delta, c): f(x)=c+\Delta\}$. Since, by Lemma 3.1, the interval $[c, c+\Delta]$ is not $f^{2}$-invariant there exists $x \in[c, c+\Delta]$ such that $f(x)<a_{1}$. Then we set $a_{2}=\min \left\{x \in(c, c+\Delta]: f(x)=a_{1}\right\}$ and $b=\max \left\{f(x): x \in\left[a_{2}, c+\Delta\right]\right\}$. We note that $b<c$ by the hypotheses. Now we are ready to define the map $\tilde{f}$. We take $L=\left(a_{1}, a_{2}\right)$ and $K \subset\left(c, a_{2}\right)$. We also take $d \in(c, \min K)$ and $d^{\prime} \in\left(\max K, a_{2}\right)$. Then we define $\tilde{f} \in \mathcal{C}(I, I)$ satisfying conditions (i) and (ii), $\tilde{f}(x)=\tilde{f}\left(a_{1}\right)=c+\Delta$ for each $x \in\left[a_{1}, b\right], \tilde{f}(x)=\tilde{f}(\min K)$ for each $x \in[d, \min K], \tilde{f}\left(d^{\prime}\right)=c$ and $\tilde{f}$ is affine on each of the intervals $[b, c],[c, d],\left[\max K, d^{\prime}\right]$ and $\left[d^{\prime}, a_{2}\right]$.

We note that the intervals $\left[c, d^{\prime}\right]$ and $K$ are $\tilde{f}$-invariant and that $\{c\}$ is the only periodic orbit lying in $\left[c, d^{\prime}\right] \backslash K$. Further, it is easy to see that there is a periodic orbit $\left\{p_{1}, p_{2}\right\}$ of $\tilde{f}$ of period 2 such that $p_{1} \in(b, c)$ and $p_{2} \in\left(d^{\prime}, a_{2}\right)$. On the other hand, if $x \in\left(d^{\prime}, a_{2}\right)$ is a periodic point of $\tilde{f}$ such that $\operatorname{Orb}(\tilde{f}, x) \subset(b, c) \cup\left(d^{\prime}, a_{2}\right)$ then, since $\tilde{f}\left(\left[d^{\prime}, a_{2}\right]\right)=\left[a_{1}, c\right]$ and $\tilde{f}\left(\left[a_{1}, c\right]\right)=[c, c+\Delta]$, we have that $x$ has even period. So, $x$ is a periodic point of $\tilde{f}^{2}$. But, since the only such point is $p_{2},\left\{p_{1}, p_{2}\right\}$ is the only periodic orbit of $\tilde{f}$ contained in $(b, c) \cup\left(d^{\prime}, a_{2}\right)$.

To end the proof of the lemma in this subcase it is enough to prove that if $P$ is a periodic orbit of $\tilde{f}$ intersecting $\left(a_{1}, c\right) \cup\left(d^{\prime}, a_{2}\right)$ then $P=\left\{p_{1}, p_{2}\right\}$. Take such an orbit. Assume first that $P \subset\left[a_{1}, c\right) \cup\left(d^{\prime}, c+\Delta\right]$. Since $f(x) \leqslant c+\Delta$ for each $x \in\left[a_{1}, c\right]$, $f(c+\Delta) \leqslant b<c$ and $f^{2}(c+\Delta)>c+\Delta$ (see Lemma 3.1) we get that $\bar{f}(c+\Delta)=$ $f(c+\Delta)<a_{1}$. Thus, $c+\Delta \notin P$. Hence, $P$ cannot contain any point from $\left[a_{1}, b\right]$ since all such points are mapped by $\tilde{f}$ to the point $c+\Delta$. Further, $P$ cannot contain any point from $\left[a_{2}, c+\Delta\right)$ since all such points are mapped by $\tilde{f}$ either into $\left[0, a_{1}\right)$ or into 
$\left[a_{1}, b\right]$. Therefore, if $P \subset\left[a_{1}, c\right) \cup\left(d^{\prime}, c+\Delta\right]$ then $P \subset(b, c) \cup\left(d^{\prime}, a_{2}\right)$ and coincides with $\left\{p_{1}, p_{2}\right\}$. Now we assume that $P \not \subset\left[a_{1}, c\right) \cup\left(d^{\prime}, c+\Delta\right]$. Since $\left[c, d^{\prime}\right]$ is $\tilde{f}$-invariant, this means that $P$ intersects $I \backslash\left[a_{1}, c+\Delta\right]$. Then, $P$ must contain a point $z \in\left(a_{2}, c+\Delta\right]$ such that $\tilde{f}(z)=f(z)<a_{1}$. Therefore, $\tilde{f}(z) \geqslant c-\delta_{1}$ because $f([c-\Delta, c+\Delta]) \subset$ $\left[c-\delta_{1}, c+\delta_{1}\right]$. Moreover, by the hypotheses we have that $f^{2}\left(\left[c-\Delta, a_{1}\right]\right) \subset[c-$ $\left.\delta_{1}, a_{1}\right]$. Also, by Lemma 3.1 we have that $\operatorname{Orb}\left(f^{2},\left[c-\delta_{1}, c-\Delta\right]\right) \subset[0, c-\Delta]$. Hence, $\operatorname{Orb}\left(f^{2},\left[c-\delta_{1}, a_{1}\right]\right) \subset\left[0, a_{1}\right]$. Consequently, $f^{2 n+1}(z) \leqslant a_{1}$ for each $n \geqslant 0$. Moreover, since $f(x)>a_{1}$ for each $x \in L$, we see that $f^{2 n}(z) \notin L$ for each $n \geqslant 0$. Thus, $\operatorname{Orb}(f, z) \subset I \backslash L$ and so, $P=\operatorname{Orb}(\tilde{f}, z)=\operatorname{Orb}(f, z)$ which contradicts the fact that $P$ intersects $\left(a_{1}, c\right) \cup\left(d^{\prime}, a_{2}\right) \subset L$.

Now we are left with the case $c=1$. The proof of this case follows similarly to the proof of the case $c \neq 1$ with all inequalities reversed.

Proof of THEOREM B: Let $F(x, y)=\left(f(x), g_{x}(y)\right)$ be a continuous triangular map of type at most $2^{\infty}$ and let $\varepsilon>0$. To prove the theorem it suffices to find a continuous triangular map $\tilde{F}(x, y)=\left(\tilde{f}(x), \tilde{g}_{x}(y)\right)$ of type $2^{\infty}$ with infinite topological entropy such that $d_{2}(F, \tilde{F})<\varepsilon$.

To construct this map we proceed as follows. Take a fixed point $c$ of $f$ and $\eta>0$ such that $d_{1}\left(g_{x}, g_{c}\right)<\varepsilon / 2$ for each $x \in[c-\eta, c+\eta] \cap I$.

Let $\phi(x, y)=\left(\varphi(x), \psi_{x}(y)\right)$ be the triangular map of type $2^{\infty}$ with infinite topological entropy from the proof of Theorem A (recall that $\varphi$ is of type $2^{\infty}$ ). Now, let $\tilde{f}$ be the map given by Lemma 3.2 with $\varphi$ instead of $g$. Since $\varphi$ is of type $2^{\infty}$, by Lemma 3.2 (ii)-(iv), $\tilde{f}$ is of type $2^{\infty}$. Now we shall define the maps $\tilde{g}_{x}$ for each $x \in I$. We consider the following cases (we use the notation from Lemma 3.2):

CASE 1. $x \in I \backslash L$. We set $\tilde{g}_{x}=g_{x}$. Then, by virtue of Lemma 3.2 (iii), each periodic orbit of $\widetilde{F}$ intersecting $(I \backslash L) \times I$ is contained in $(I \backslash L) \times I$ and, hence, by Lemma 3.2 (i) it has period a power of two because $F$ (and $f$ ) is of type at most $2^{\infty}$.

CASE 2. $\boldsymbol{x}$ is a periodic point of $\tilde{f}$ in $L \backslash K$. We set $\tilde{g}_{x}=g_{c}$. Then, since $g_{c}$ is of type at most $2^{\infty}$ (recall that $c$ is a fixed point of $f$ ), by Lemma 3.2 (iv) we get that the map $\tilde{F}$ will have no periodic points of period different from a power of two in $I_{x}$.

CASE 3. $x \in K$. By Lemma 3.2 (ii) $\left.\widetilde{f}\right|_{K}$ is topologically conjugate to $\varphi$. Thus, there exists a homeomorphism $\xi: I \rightarrow K$ such that $\left.\tilde{f}\right|_{K} \circ \xi=\xi \circ \varphi$. Now take $\tilde{c}$, a fixed point of $g_{c}$, and use Lemma 3.2 with $g_{c}, \psi_{\xi^{-1}(x)}$ and $\varepsilon / 2$ instead of $f, g$ and $\varepsilon$ to obtain the map $\tilde{g}_{x}$ and intervals $\widetilde{K} \subset \widetilde{L}$ satisfying all conditions from the statement of the lemma (in particular we have $d_{1}\left(\tilde{g}_{x}, g_{c}\right)<\varepsilon / 2$ ). From the proof of Lemma 3.2 it is clear that the intervals $\widetilde{K}$ and $\tilde{L}$ depend only on the map $g_{c}$, not on $x$. Moreover, since $\psi_{z}(0)=0$ and $\psi_{z}(1)=1$ for each $z \in I$, we see that $\left.\widetilde{g}_{x}\right|_{I \backslash \widetilde{K}}$ also is independent 
of $x$. Since $g_{c}$ is of type at most $2^{\infty}$, by Lemma 3.2 (i)-(iv) we get that $\left.\tilde{g}_{x}\right|_{I \backslash \tilde{K}}$ has no periodic point with period different from a power of two. Therefore, since $\left.\tilde{f}\right|_{K}$ is topologically conjugate to $\varphi$ which has type $2^{\infty},\left.\tilde{F}\right|_{K \times(I \backslash \tilde{K})}$ has type at most $2^{\infty}$. On the other hand, $\left.\widetilde{F}\right|_{K \times \tilde{K}}$ is topologically conjugate to $\Phi$. Thus, $\widetilde{F}$ has infinite topological entropy and $\left.\tilde{F}\right|_{K \times \tilde{K}}$ is of type $2^{\infty}$.

CASE 4. $x \in L \backslash K$ but it is not a periodic point of $\tilde{f}$. Note that $\tilde{F}$ has no periodic point in $I_{x}$ independently of the choice of $\tilde{g}_{x}$ for all $x \in I$. So, for $x \in I$, we let $\tilde{g}_{x}$ be an arbitrary one-parameter family of continuous maps in $I$, depending continuously on $x$, such that $d_{1}\left(\tilde{g}_{x}, g_{c}\right)<\varepsilon / 2$ for each $x \in L \backslash K$ which is not a periodic point of $\tilde{f}$. Such a family exists because $\tilde{g}_{c}=g_{c}, c \in L$, and for each $z \in L$ for which $\tilde{g}_{z}$ has been defined we already have $d_{1}\left(\tilde{g}_{z}, g_{c}\right)<\varepsilon / 2$ and the same is true for each $z \in[c-\eta, c+\eta] \cap(I \backslash L)$.

From all said above we have a map $\tilde{F}$ with infinite topological entropy and type $2^{\infty}$. To end the proof only it remains to show that $d_{2}(F, \tilde{F})<\varepsilon$. To prove this we note that, by Lemma $3.2(v)$ we have $d_{1}(f, \tilde{f})<\varepsilon$. On the other hand, $d_{1}\left(g_{x}, \tilde{g}_{x}\right)=0$ for each $x \in I \backslash L$. For $x \in L \subset[c-\eta, c+\eta]$ we have $d_{1}\left(g_{x}, g_{c}\right)<\varepsilon / 2$ and $d_{1}\left(g_{c}, \tilde{g}_{x}\right)<\varepsilon / 2$. Hence, $d_{1}\left(g_{x}, \tilde{g}_{x}\right)<\varepsilon$ for all $x \in I$ and, thus, $d_{2}(F, \tilde{F})<\varepsilon$.

\section{Proof of Theorem C}

The idea of the proof is similar to the one used in [17] in the proof that, in $\mathcal{C}_{\Delta}\left(I^{2}, I^{2}\right)$, the Sharkovskir ordering is not stable in the sense of Block.

Proof of Theorem C: For $\lambda \in[0,1]$ let $F_{\lambda}:(x, y) \mapsto(\lambda x, x g(y))$ where $g \in$ $\mathcal{C}(I, I)$ is any map with positive, say infinite, entropy. Since $h\left(\left.F_{1}\right|_{I_{1}}\right)=h(g)=\infty$, we have $h\left(F_{1}\right)=\infty$. On the other hand, for $\lambda<1$ we have $h\left(F_{\lambda}\right)=h\left(\left.F_{\lambda}\right|_{I_{0}}\right)=0$.

\section{LOWER BOUNDS OF THE TOPOLOGICAL ENTROPY FOR TRIANGULAR MAPS}

In this Section we prove Theorem D. We start with some remarks and auxiliary results.

REMARK 5.1. It is not difficult to see, (see for instance [3]) that if $n<, k$ then $\tau_{n} \leqslant \tau_{k}$. Moreover, if $k$ is not a power of two then $\tau_{n}<\tau_{k}$.

The following technical lemma on the numbers $\tau_{n}$ will be useful later.

Lemma 5.2. For each $m \geqslant 0$ and $s \in \mathbb{N}$ we have

$$
2^{-m} \log \tau_{s}=\log \tau_{2^{m}}
$$


ProOF: Let $s=2^{j} q$ with $j \geqslant 0$ and $q \geqslant 1$ odd. If $q=1$ then $2^{-m} \log \tau_{s}=0=$ $\log \tau_{2^{m+j}}=\log \tau_{2^{m}}$. If $q>1$ then $2^{-m} \log \tau_{a}=2^{-m} \log \left(\lambda_{q}\right)^{2^{-j}}=\log \left(\lambda_{q}\right)^{2^{-(m+j)}}=$ $\log \tau_{2^{m+j}}=\log \tau_{2^{m}}$.

The following proposition gives the lower bounds of the topological entropy for continuous self-maps of the interval in terms of the set of periods (see $[5,12,13])$.

Proposition 5.3. Let $f \in \mathcal{C}(I, I)$ have a periodic orbit of period $n$. Then $h(f) \geqslant \log \tau_{n}$. Conversely, for each $n \in \mathbb{N}$ there exists a map $f_{n} \in \mathcal{C}(I, I)$ of type $n$ such that $h\left(f_{n}\right)=\log \tau_{n}$.

Proof of Theorem D: As usual, set $F(x, y)=\left(f(x), g_{x}(y)\right)$. Denote a periodic orbit of $F$ of period $n$ by $P$. Since $F$ is triangular we know that $\operatorname{pr}(P)$ is a periodic orbit of $f$ of period $l$ and $l$ divides $n$.

Assume that $l$ is not a power of two. Then, clearly, $n \leqslant l$. Therefore, by Remark 5.1 and Proposition 5.3 we get

$$
h(F) \geqslant h(f) \geqslant \log \tau_{l} \geqslant \log \tau_{n} .
$$

Assume now that $l$ is a power of two and let $x \in \operatorname{pr}(P)$. Then, $I_{x}$ is $F^{l}$-invariant and $\left.F^{l}\right|_{I_{x}}$ has a periodic orbit of period $s=n / l$. Therefore, by Proposition 5.3 and Lemma 5.2 we get

$$
h(F)=\frac{1}{l} h\left(F^{l}\right) \geqslant \frac{1}{l} h\left(\left.F^{l}\right|_{I_{x}}\right) \geqslant \frac{1}{l} \log \tau_{\lrcorner}=\log \tau_{l,}=\log \tau_{n} .
$$

Now we prove the converse. We define $F_{n} \in \mathcal{C}_{\Delta}\left(I^{2}, I^{2}\right)$ by $F_{n}(x, y)=\left(f_{n}(x), y\right)$, where $f_{n}$ is the interval map from the statement of Proposition 5.3. It is not difficult to show that $h_{f_{n}}\left(F_{n}\right)=0$. Therefore we get

$$
h\left(F_{n}\right)=h\left(f_{n}\right)=\log \tau_{n}
$$

Furthermore, by the definition of $F_{n}$ we have $\operatorname{Per}\left(F_{n}\right)=\operatorname{Per}\left(f_{n}\right)$ and so $F_{n}$ is of type $n$.

REMARK 5.4. From the proof of the above theorem we see that if the periodic orbit of $F$ of period $n$ under consideration projects on a periodic orbit of $f$ with period $l$ such that $l$ is not a power of two then, in fact, we have $h(F) \geqslant \log \tau_{l}$.

In view of Remark 5.1, if $l \neq n$, this lower bound of the topological entropy is strictly larger than the one given by Theorem D. Moreover, if $n=2^{m} q$ with $m>0$ and $q>1$ odd then there exists a triangular map of the form $F(x, y)=(f(x), g(y))$ having a periodic orbit of period $n$ whose projection is a periodic orbit of $f$ of period $q$ and such that $h(F)=\log \tau_{q}>\log \tau_{n}$. The construction of this map is as follows. 
We take as $f$ the map $f_{q}$ from Proposition 5.3 and as $g$ the map $f_{2^{m}}$ also from Proposition 5.3. Let $Q$ be the periodic orbit of period $q$ of $f$ and let $R$ be the periodic orbit of period $2^{m}$ of $g$. Since $q$ and $2^{m}$ are relatively prime we have that $Q \times R$ is a periodic orbit of $F$ of period $n$. Since

$$
0=\log \tau_{2^{m}}=h(g)=h(g, I)=\lim _{\varepsilon \rightarrow 0} \limsup _{n \rightarrow \infty} \frac{1}{n} \log s_{n}(\varepsilon, I, g)
$$

we obtain that $h\left(F, I_{x}\right)=0$ for all $x \in I$. Therefore, $h_{f}(F)=0$ and, hence,

$$
h(F)=h(f)=\log \tau_{q}
$$

\section{REFERENCES}

[1] R. Adler, A. Konheim and J. McAndrew, 'Topological entropy', Trans. Amer. Math. Soc. 114 (1965), 309-319.

[2] L. Alsedà, J. Llibre and M. Misiurewicz, Combinatorial dynamics and entropy in dimension one (World Scientific, Singapore, 1993).

[3] L. Alsedà and J.M. Moreno, 'Lower bounds of topological entropy of maps of $Y^{\prime}$, J. Math. Anal. Appl. 155 (1991), 512-530.

[4] L. Block and W.A. Coppel, Dynamics in one dimension, Lecture Notes in Math. 1513 (Springer-Verlag, Berlin, Heidelberg, New York, 1992).

[5] L. Block, J. Guckenheimer, M. Misiurewicz and L.S. Young, 'Periodic points and topological entropy of one dimensional maps', in Global theory of dynamical systems, Lecture Notes in Math. 819 (Springer-Verlag, Berlin, Hedelberg, New York, 1980), pp. 18-34.

[6] A.M. Blokh, 'On some properties of graph maps; spectral decomposition, Misiurewicz conjecture and abstract sets of periods', Preprint \#35, Max-Plank-Institut für Mathematik.

[7] R. Bowen and J. Franks, 'The periodic points of maps of the disk and the interval', Topology 15 (1976), 337-342.

[8] R. Bowen, 'Entropy for group endomorphisms and homogeneous spaces', Trans. Amer. Math. Soc. 153 (1971), 401-414.

[9] R. Bowen, 'Periodic points and measures for axiom $A$ diffeomorphisms', Trans. Amer. Math. Soc. 154 (1971), 377-397.

[10] E.I. Dinaburg, 'Connection between various entropy characterizations of dynamical systems', (Russian), Izv. Akad. Nauk. SSSR 35 (1971), 324-366.

[11] J.M. Gambaudo, S. van Strien and C. Tresser, 'The periodic orbit structure of orientation preserving diffeomorphisms on $D^{2}$ with topological entropy zero', Ann. Inst. H. Poincaré Phys. Théor. 49 (1989), 335-356.

[12] L. Jonker and D. Rand, 'The periodic orbits and entropy of certain maps of the unit interval', J. London Math. Soc. 22 (1980), 175-181.

[13] L. Jonker and D. Rand, 'Une borne inferieure pour l'entropie de certaines applications de l'intervalle dans lui-mème', C.R. Acad. Sci., Paris, Sér. I Math. 287 (1978), 501-502. 
[14] P.E. Kloeden, 'On Sharkovsky's cycles coexistence ordering', Bull. Austral. Math. Soc. 20 (1979), 171-177.

[15] S.F. Kolyada, 'On triangular maps of type $2^{\infty}$ with positive entropy', (Russian), in $D y$ namical systems and turbulence, Inst. Math. Ukrain. Acad. Sci. Kiev., 1989, pp. 76-82.

[16] S.F. Kolyada, 'On dynamics of triangular maps of the square', Preprint 91.14. Inst. Math. Ukrain. Acad. Sci., Kiev, 1991, Ergodic Theory and Dynamical Systems (to appear).

[17] S.F. Kolyada and A.N. Sharkovsky, 'On topological dynamics of triangular maps of the plane', in Proc. ECIT-89 (World Scientific, Singapore, 1991), pp. 177-183.

[18] T.Y. Li, M. Misiurewicz, G. Pianigiani and J.A. Yorke, 'No division implies chaos', Trans. Amer. Math. Soc. 273 (1982), 191-199.

[19] J. Llibre and R.S. Mackay, 'A classification of braid types for diffeomorphisms of surfaces of genus zero with topological entropy zero', J. London Math. Soc. (2) 42 (1990), 562-576.

[20] J. Libre and M. Misiurewicz, 'Horseshoes, entropy and periods for graph maps', Topology (to appear).

[21] M. Misiurewicz, 'Horseshoes for mappings of an interval', Bull. Acad. Pol. Sci., Ser. Sci. Math. 27 (1979), 167-169.

[22] A.N. Sharkovsky, 'Coexistence of cycles of a continuous map of the line into itself, (Russian), Ukrain. Math. Zh. 16 (1964), 61-71.

[23] A.N. Sharkovsky, S.F. Kolyada, A.G. Sivak and V.V. Fedorenko, 'Dynamics of one- dimensional maps', (Russian), Naukova Dumka. Kiev (1989).

Departament de Matemàtiques

Universitat Autònoma de Barcelona

08193 - Bellaterra

Barcelona

Spain

Department of Mathematics

Faculty of Education

Tajovského 40

97549 Banská Bystrica

Czechoslovakia
Institute of Mathematics

Ukrainian Academy of Sciences

Repin str.3

$252601 \mathrm{Kiev}-4$

Ukraine 